Document downloaded from:

http://hdl.handle.net/10251/82091

This paper must be cited as:

Fuentes Muela, M.; Gimenez Gandia, JJ.; Gómez Barquero, D. (2016). Optimization and Performance of Non-Uniform Rotated Constellations With Multi-RF Transmission Technique. IEEE Transactions on Broadcasting. 62(4):855-863. doi:10.1109/TBC.2016.2576601.

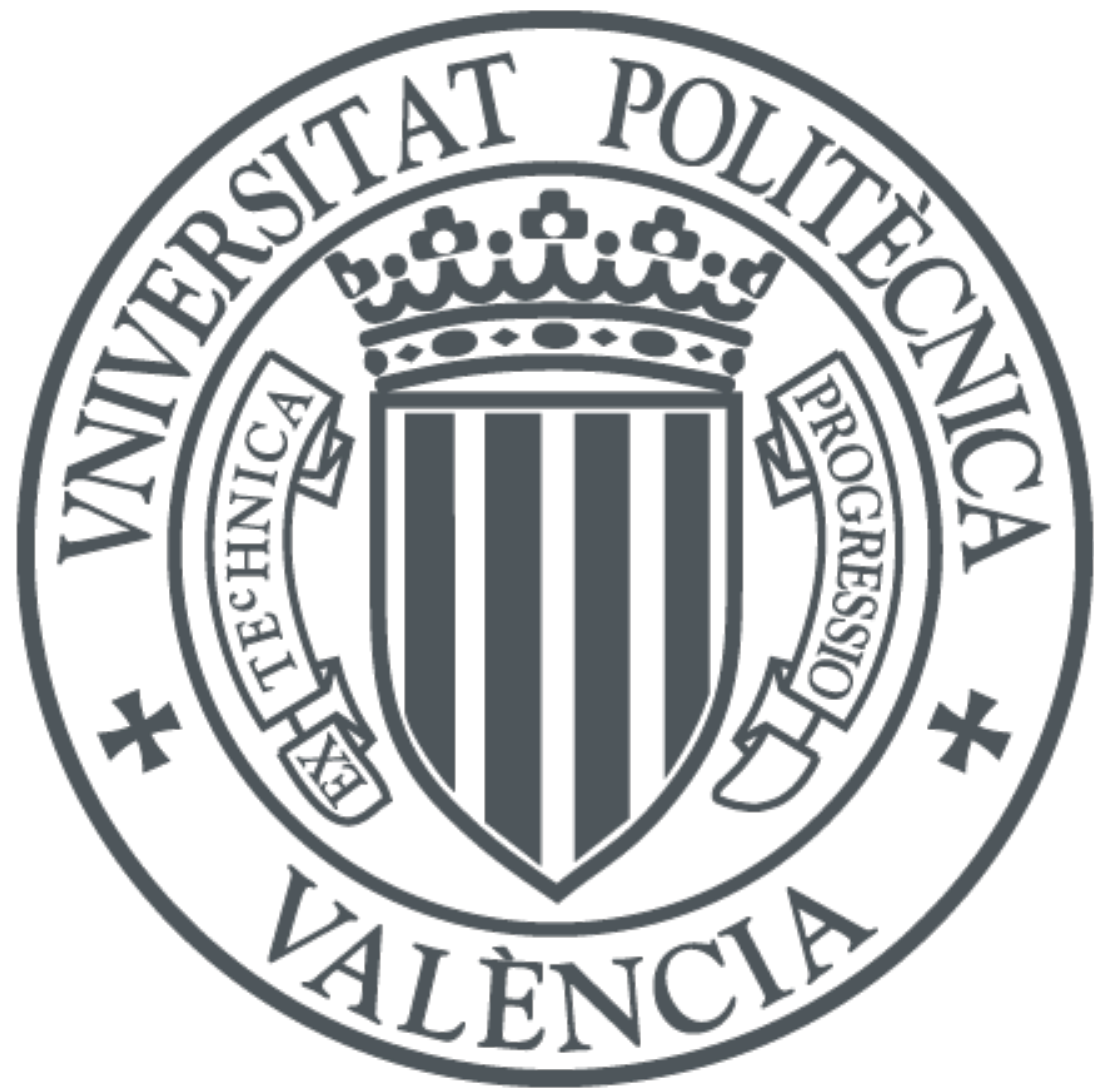

The final publication is available at

http://dx.doi.org/10.1109/TBC.2016.2576601

Copyright Institute of Electrical and Electronics Engineers (IEEE)

Additional Information

"(c) 2016 IEEE. Personal use of this material is permitted. Permission from IEEE must be obtained for all other users, including reprinting/ republishing this material for advertising or promotional purposes, creating new collective works for resale or redistribution to servers or lists, or reuse of any copyrighted components of this work in other works.") 


\title{
Optimization and Performance of Non-Uniform Rotated Constellations with Multi-RF Transmission Techniques
}

\author{
Manuel Fuentes, Jordi Joan Giménez, and David Gómez-Barquero
}

\begin{abstract}
Non-Uniform Constellations (NUC) have been introduced in ATSC 3.0 (Advanced Television Systems Committee Third Generation) as one of the main novelties to improve the performance compared to uniform Quadrature Amplitude Modulation (QAM) constellations. NUCs are optimized by means of signal geometrical shaping, considering the signal-to-noise ratio (SNR) and the channel model. ATSC 3.0 implements two types of NUC, depending on the number of real-valued dimensions in which they are optimized: one-dimensional and two-dimensional NUCs (1D-NUC and 2D-NUC, respectively). However, the gain of NUCs becomes almost non-existent at high SNRs, especially when optimizing for fading channels. In that particular case, Rotated Constellations (RC) can be used to further improve the overall system performance. RCs may become especially effective when using multi-radio frequency (multi-RF) SNR averaging techniques such as Channel Bonding $(\mathrm{CB})$ or Time-Frequency Slicing (TFS), where in-phase (I) and quadrature (Q) components are transmitted in different RF channels. 2D-NUCs can be rotated without increasing the demapping complexity, since a 2D-demapper is also needed. In this paper, we propose an optimization method designed for rotated 2D-NUCs, in which the rotation angle is considered as an additional variable, together with the symbol positions. The SNR gain obtained in fading channels is also provided for three different use cases: single-RF transmissions, CB with 2 RF channels as adopted in ATSC 3.0, and extension of multi-RF techniques to 4 RF channels.
\end{abstract}

Index Terms-Non-Uniform Constellations, Rotated Constellations, Channel Bonding, ATSC 3.0, Time Frequency Slicing, DTT.

\section{INTRODUCTION}

$\mathbf{N}$ ON-UNIFORM Constellations (NUC) have been introduced in new-generation Digital Terrestrial Television (DTT) systems to reduce the gap between the BICM (BitInterleaved Coding and Modulation) capacity of uniform Gray-labelled constellations and the unconstrained Shannon limit [1], [2]. This difference to the capacity limit also increases with the modulation order. With uniform Quadrature Amplitude Modulation (QAM) constellations, the symbols are regularly spaced in the constellation diagram, i.e. with the same distance among symbols in both in-phase (I) and quadrature (Q) components. Uniform QAM constellations have been widely used in many broadcast systems currently deployed worldwide, such as ISDB-T (Integrated Services

This work was partially supported by the Ministry of Economy and Competitiveness of Spain (TEC2014-56483-R), co-funded by the European FEDER fund.

The authors are with the Institute of Telecommunications and Multimedia Applications (iTEAM) of the Universtat Politecnica de Valencia, 46022 Valencia, Spain, (e-mail: \{mafuemue, jorgigan, dagobar\}@iteam.upv.es).

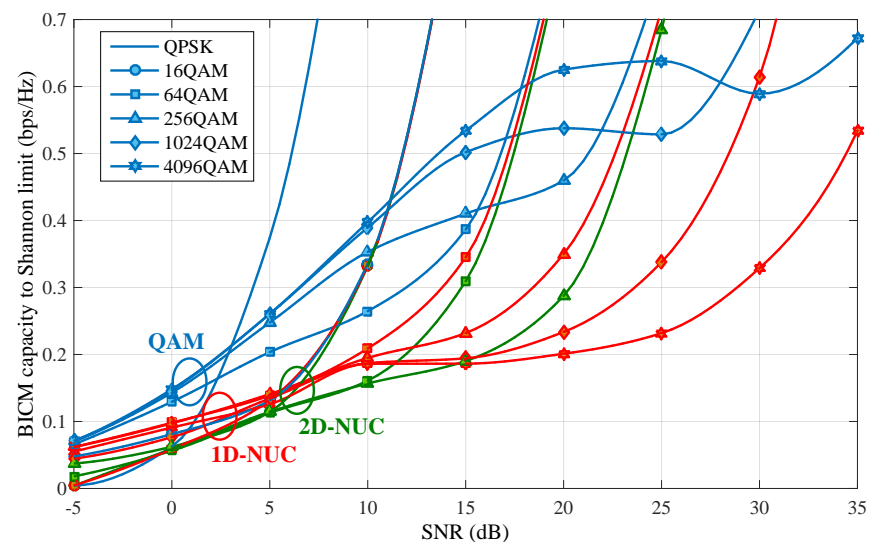

Fig. 1. Shortfall of the BICM capacity from the unconstrained Shannon capacity, for the i.i.d. Rayleigh channel and a range of Gray-mapped QAM and 1D/2D-NUC constellations.

Digital Broadcasting - Terrestrial), [3], DVB-T (Digital Video Broadcasting - Terrestrial) [4] or DVB-T2 (Terrestrial 2nd Generation) [5]. However, the distribution of the constellation symbols is not optimum, due to the two constraints imposed by assuming a rectangular shape and equally spaced levels. With NUCs, the constellation symbols are optimized by means of signal shaping techniques to provide an improved performance compared to uniform QAM constellations.

There are two types of NUCs, depending on the number of real-valued dimensions considered in the optimization process: one-dimensional and two-dimensional NUCs (1D-NUC and 2D-NUC, respectively) [6]. ATSC 3.0 (Advanced Television Systems Committee - Third Generation) is the first standard that includes 2D-NUCs from 16NUC to 256NUC orders, and 1D-NUCs for new high-orders such as 1024NUC (or 1kNUC) and 4096NUC (or 4kNUC) [7]. 1D-NUCs have a squared shape with non-uniform distance between the constellation symbols [8]. 2D-NUCs are designed by relaxing the square shape constraint, with a better performance than 1D-NUCs but with a higher receiver complexity since they cannot be separated into two independent I/Q components [9]. Fig. 1 shows the BICM capacity to the Shannon limit given by uniform QAM vs. optimized NUCs. In this case, all 1Dand 2D-NUCs (including 1D-16NUC, 1D-64NUC and 1D256NUC, which are not specified in ATSC 3.0) have been properly optimized for a range of signal-to-noise ratios (SNR) from -5 to $35 \mathrm{~dB}$, for i.i.d. (independent and identically 
distributed) Rayleigh channel.

Previous works in the literature outline the design of NUCs and their potential gains. Reference [10] noted the capacity shortfall for uniform QAMs, and introduced the non-uniform concept, obtaining several constellations which offer a capacity improvement. A more recent study [11] tackles the optimization of 1D-NUCs, in an Additive White Gaussian Noise (AWGN) channel. In [12], high-order 1D- and 2D-NUCs are optimized with respect to their BICM capacity, also for AWGN channel. In [13], high-order NUCs with constellation sizes of up to 4k-QAM are investigated. A sub-optimal demapper that reduces the total number of distances to be computed when using 2D-NUCs has also been proposed in [14].

The gain of NUCs becomes almost non-existent at high SNRs, especially when optimizing for fading channels [6], [12]. In this particular case, Rotated Constellations (RC) can be used to improve the overall system performance [15], without increasing the demapper complexity. With RCs, a certain rotation angle is applied to the constellation, so that the binary information is transmitted simultaneously in different I and Q components. In order to ensure that each component undergoes independent fading, a component interleaver (CIL) is applied after the rotation to separate both components in time and frequency domains. Thus, it is possible to retrieve all the information at the receiver side even when one of the components has been erased by the channel. With NUCs, it is required to optimize different rotation angles. Rotation angles are optimized so that the diversity experimented by both I and $\mathrm{Q}$ components is maximized.

In ATSC 3.0, constellation rotation was not adopted. In a single radio frequency ( $R F$ ) transmission, the rotation gain is only significant for some particular combinations, i.e. low order constellations and high code rates (CR) [14]. However, RCs may become especially effective when using multi-RF techniques, such as Channel Bonding (CB) [16], providing very high performance gains. CB has been adopted in ATSC 3.0 and consists of splitting service data across two RF channels, so that peak data rate can be doubled. In such case, it is desirable that each component is transmitted in a different RF channel using a proper CIL, which must be redesigned. Results can be also extended to any multi-RF technology, such as Time-Frequency Slicing (TFS) [17]. TFS was proposed in DVB-T2 and adopted in DVB-NGH (NextGeneration Handheld) [2]. With TFS the data is transmitted in a slot-by-slot manner by frequency hopping across an RF-Mux of two or more RF channels (in practice, up to 6).

The use of Non-Uniform Rotated Constellations (NURC) has not yet been studied in connection with multi-RF techniques where they may become a good solution to increase robustness. In this paper, we propose an optimization method designed for rotated 2D-NUCs, in which the rotation angle is considered as an additional variable. Afterwards, we first provide the rotation gain in single-RF transmissions. We also analyze NURCs with multi-RF technologies for 2 and 4 RF channels, observing the performance gain compared to nonrotated NUCs and single-RF transmissions.

The rest of the paper is structured as follows. Section II describes the two proposed optimization methods of NURCs
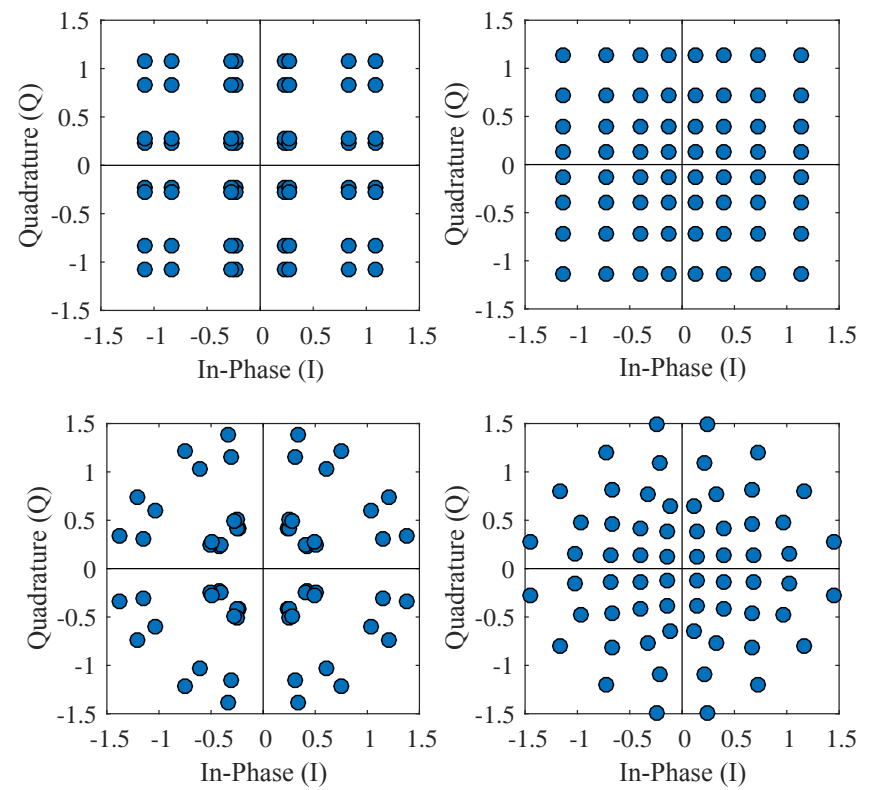

Fig. 2. Examples of optimized constellations: 1D-NUCs for a low SNR of 5 $\mathrm{dB}$ (top left) and a high SNR of $12 \mathrm{~dB}$ (top right), 2D-NUCs for a low SNR of $5 \mathrm{~dB}$ (bottom left) and a high SNR of $12 \mathrm{~dB}$ (bottom right).

for fading channels. In Section III, the basics of multi$\mathrm{RF}$ techniqes are explained, and the designed CIL is also described. In Section IV, performance results of optimized NURCs are shown, applied to several scenarios, i.e. in a single $\mathrm{RF}$ transmission, or combined with multi-RF techniques. Finally, the main findings of the work are summarized in Section V.

\section{NON-UNiform RotATED CONSTELlATIONS OPTIMIZATION}

The improved performance when using NUCs comes from an optimization of the constellation symbols so that the BICM capacity is maximized [12]. With fading channels, the average of the BICM capacity of the channel is the target function of the optimization. Hence, each target SNR requires a different optimized NUC. In addition, the transmitted symbols need to fulfil a specific constraint. The power of the symbol alphabet has to be normalized to unity. Note that when considering performance simulations with strong error correcting codes such as Low-Density Parity Codes (LDPC), the target SNR of the NUC is selected for each CR according to the SNR of the waterfall region. In this paper, we use CRs available in the ATSC 3.0 specification, i.e. from $2 / 15$ to $13 / 15$, with step $1 / 15$ [18].

When optimizing NUCs at low SNRs, they collapse into constellations with a lower order, where almost identical symbols are grouped in clusters. We call these constellations condensed constellations. With the condensation, the most significant bits (MSB) provide similar robustness as the positions of low-order constellations. On the other hand, the least significant bits (LSB) cannot be resolved from the overlapping points, since they offer very weak information. At high SNRs, the positions converge toward uniform values. Without a robust 
$\mathrm{CR}$, the best option is to pack the constellation symbols as uniformly spaced as possible. The tendency can be found in Fig. 2, where we show different 1D- and 2D-NUCs designed for low and high SNRs.

Packing the symbols in almost uniform distributions implies very low NUC gains compared to QAM, especially when optimizing for fading channels. In that particular case, RCs can be used to improve the overall system performance. When applying an additional rotation to the constellation, there are two different possibilities. The simplest way is to optimize first the constellation symbols and apply afterwards a rotation, at the expense of a penalty loss (constellations are optimized without including the rotation). We call this method Optimization Before Rotation (OBR). As an improved solution, we propose to include the rotation angle as a new variable in the optimization process. This second method is called Optimization with Additional Rotation (OAR). Both methods are explained next.

\section{A. Optimization Before Rotation (OBR)}

1) Non-Uniform Constellations Optimization: The total degrees of freedom (DOF) in the 1D-NUC optimization are $\frac{\sqrt{M}}{2}-1$, where $M$ represents the number of symbols of the constellation under evaluation. The aim with 1D-NUCs is to identify the optimum parameters $\mathbf{a}=\left[a_{0}, a_{1}, \ldots, a_{N}\right]$ that indicate the symbol values in a specific component I or $\mathrm{Q}$ (real or imaginary part, respectively), where $N$ represents the total DOF. Due to the constraint of power normalization, the smallest value $a_{0}$ is set to 1 . For an SNR target, the BICM capacity is calculated as a function of $\mathbf{a}$. For instance, assuming a uniform 16QAM with positions $\{-3,-1,+1,+3\}$ on each axis, then it is possible to optimize a $1 \mathrm{D}-16 \mathrm{NUC}$ with positions $\left\{-a_{1},-1,+1,+a_{1}\right\}$, using a single parameter $a_{1}$. Optimizing higher order constellations requires to increase the number of variables, which also increments the optimization burden. The number of optimization parameters for constellations with cardinality of 64, 256, 1024, and 4096 points, require: 3, 7, 15 and 31 optimization variables, respectively. Note that with QPSK the DOF is 0, and there is no possible optimization with this constellation. In addition, high order constellations require optimization algorithms that reduce the complexity, due to the high number of parameters to be evaluated. In this paper, we use the Nelder-Mead (simplex) optimization algorithm [19]. Fig. 2 (top) shows two examples of 1D-64NUCs optimized for SNRs of 5 and $12 \mathrm{~dB}$, for the i.i.d. Rayleigh channel.

2D-NUCs have arbitrary shape along the complex I/Q plane and, in this paper, we assume that the constellations retain left-right and up-down symmetry [12]. Due to this symmetry, the complete vector can be derived by defining just the first quarter of the complex constellation symbols, which reduces the optimization complexity. It has been proved with capacity simulations that the the improvement achieved when optimizing all constellation symbols is almost negligible. However, real and imaginary parts must be calculated separately, so the number of parameters increases significantly compared to $1 \mathrm{D}$ NUCs. The total number of DOF with 2D-NUCs is $\frac{M}{2}-1$. The number of optimization parameters for constellations with

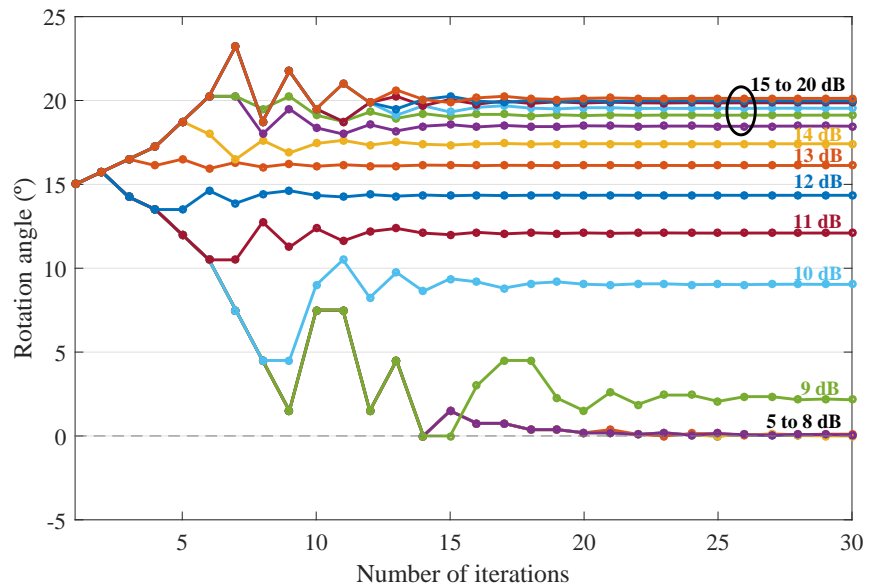

Fig. 3. Rotation angle optimization process with method OBR. 16NURC for a SNR range from 5 to $20 \mathrm{~dB}$.

cardinality of 16, 64, 256, 1024, and 4096 points, require: 7 , $31,127,511$ and 2047 optimization variables, respectively. Due to the high complexity, we only consider 2D-NUCs up to 256 symbols [14]. Fig. 2 (bottom) shows two examples of 2DNUCs optimized for SNRs of 5 and $12 \mathrm{~dB}$, for i.i.d. Rayleigh channel.

2) Rotation Angles Search: Generally speaking, with rotated constellations the demapper has to consider all symbols in both dimensions, regardless of the constellations shape. Comparing 1D- and 2D-NURCs, it is better to use 2D-NURCs, as they always provide the best capacity for a given SNR (see Fig. 1). In addition, the rotation of 2D-NUCs does not increase the demapping complexity, since a 2D-demapper is also needed. A typical 2D rotation is performed by multiplying two real (I and Q) components by an orthogonal rotation matrix of size $2 \times 2$, as shown in Eq. 1 .

$$
\left[\begin{array}{l}
\operatorname{Re}\left(y_{0}\right) \\
\operatorname{Im}\left(y_{0}\right)
\end{array}\right]=\left[\begin{array}{cc}
+a & -b \\
+b & +a
\end{array}\right]\left[\begin{array}{l}
\operatorname{Re}\left(x_{0}\right) \\
\operatorname{Im}\left(x_{0}\right)
\end{array}\right]
$$

where $x_{0}$ is the original symbol, $y_{0}$ is the resulting rotated symbol, $a=\cos \left(\frac{2 \pi \theta}{360}\right)$ and $b=\sin \left(\frac{2 \pi \theta}{360}\right)$, being $\theta$ the rotation angle in degrees. Selected angles are those that provide the maximum BICM capacity using the i.i.d. Rayleigh channel model. As an example of application, Fig. 3 shows the rotation angle optimization process for 16NURC, from an initial angle of $15^{\circ}$ (optimized for QAM constellations) and using the Nelder-Mead algorithm [19], for a SNR range from 5 to 20 $\mathrm{dB}$. The higher the SNR target, the larger the rotation angle. By contrast, when the SNR is significantly lower, the rotation angle becomes 0 , which implies better to use a non-rotated NUC.

It should be noted that RCs can be specified in two (2D) or four (4D) dimensions, depending on the number of real components in which the rotation is applied. A typical 4D rotation is performed by multiplying 4 real (two I and Q) components by an orthogonal rotation matrix of size $4 \times 4$. With a 4D rotation, two different symbols are combined. Hence, the demapping complexity is drastically increased, compared to 2D. For this reason, 4D rotation was only adopted for QPSK 

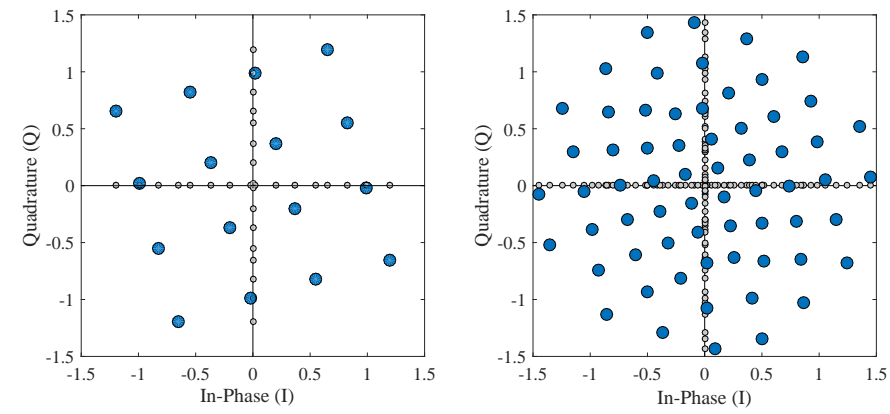

Fig. 4. 16NURC and 64NURC optimized for a SNR target of 15 and $20 \mathrm{~dB}$, and rotation angles of $18.5^{\circ}$ and $12^{\circ}$, respectively.

in the DVB-NGH specification [2]. In this paper, we only consider a $2 \mathrm{D}$ rotation with NURCs, since they require a very high demapping complexity compared with QPSK. For further information on $4 \mathrm{D}$ rotation, please refer to [15].

Fig. 4 shows two examples of 2D-NURCs optimized with the OBR method. On the left, a 16NURC optimized for a SNR target of $15 \mathrm{~dB}$, for i.i.d. Rayleigh channel, with a rotation angle of $18.5^{\circ}$. On the right, a 64NURC optimized for 20 $\mathrm{dB}$, with a rotation angle of $12^{\circ}$. For this purpose, a CIL must be implemented after the rotation. For instance, when using a single RF channel, this could be achieved by a simple time shifting of the $\mathrm{Q}$ component (Q-delay, as specified in DVB-T2), so that the Q component of symbol $n$ is transmitted with the I component of the symbol $n+1$. This ensures each component of the same symbol experiences a different fading realization.

\section{B. Optimization with Additional Rotation (OAR)}

Unlike OBR, with OAR the rotation angle is included as a new variable in the process, and the total number of DOF with 2D-NURCs is $\frac{M}{2}$. Compared to OBR, the optimization burden complexity only increases in a single DOF, and both stages are performed in a single optimization step. Moreover, the constellation symbols are optimized without capacity loss because of the rotation. As a main drawback, optimized NURCs could cause a SNR loss if the rotation is not applied in transmission.

As an example, Fig. 5 shows the capacity gain achieved for 16NURCs with both OBR and OAR methods, compared to non-rotated QAM constellations. Note that the two contributions of the OBR method are considered by separate, i.e., the NUC optimization (blue) and the following rotation (red), while the only contribution of OAR is shown in gray. Observing Fig. 5, the maximum BICM capacity is significantly higher using the second method OAR, especially for medium SNRs, region where the rotation starts to provide a slight gain (range from 9 to $11 \mathrm{~dB}$ ). In addition, optimizing NURCs with OAR increases the SNR range where there is a gain with the rotation $(7$ and $8 \mathrm{~dB})$. Thus, NURCs optimized with the OAR method are used in following sections. Table I shows the resulting rotation angles, for CRs that provide a rotation gain ( $7 / 15$ to $13 / 15$ ) higher than $0.1 \mathrm{~dB}$. A hyphen indicates that the best option is not to apply the rotation, for the particular

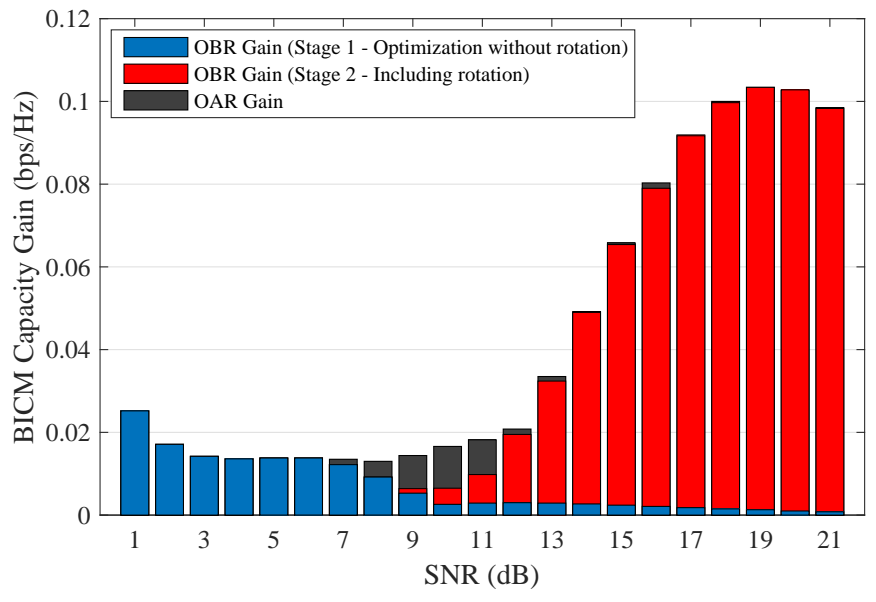

Fig. 5. BICM capacity gain of 16 NURC compared to non-rotated QAM, obtained with OBR and OAR methods. The two contributions of OBR are shown by separate.

TABLE I

ROTATION ANGLES $\left({ }^{\circ}\right)$ OPTIMIZED FOR 2D-NURCS, FOR I.I.D RAYLEIGH CHANNEL.

\begin{tabular}{|c||c|c|c|c|c|c|c|}
\hline Code Rate & $7 / 15$ & $8 / 15$ & $9 / 15$ & $10 / 15$ & $11 / 15$ & $12 / 15$ & $13 / 15$ \\
\hline \hline QPSK & 15.2 & 23.6 & 25.5 & 26.4 & 27.1 & 27.4 & 27.6 \\
\hline 16NURC & - & 8.4 & 10.3 & 14.4 & 16.1 & 18.4 & 19.5 \\
\hline 64NURC & - & - & - & - & 5.5 & 9.7 & 12.1 \\
\hline 256NURC & - & - & - & - & - & 7.5 & 10.2 \\
\hline
\end{tabular}

case of single-RF transmissions, since the gain obtained is almost negligible. As can be observed, the optimum rotation angle is higher for low-order constellations and high CRs. Rotation gains obtained using the selected angles are provided in Section IV.

\section{Application of NURCs to Multi-RF Techniques}

TFS distributes the data of each service across multiple $\mathrm{RF}$ channels by means of time slicing. Data is received by means of frequency hopping [17]. On the other hand, CB consists of the reception of data in parallel from two RF channels and enables doubling peak service data rate. There are two different operation modes. The basic mode is known as plain $\mathrm{CB}$, in which reception is performed by means of two tuners. The second operation mode, known as SNR averaging, exploits increased frequency diveristy by means of frequency interleaving of the service data among two RF channels, thus improving transmission robustness (similar to TFS) [16]. With TFS, frequency interleaving is achieved by a time interleaving duration that covers the transmission over multiple RF channels. With CB and SNR averaging, a cell exchanger is used so that one half of data is sent over each RF channel (see Fig. 6). The symbols are then received using an cell re-exchanger. Afterwards, each output signal has to be sent to a different demodulator stage, where decoding process takes place.

The main advantage of TFS and CB with SNR averaging is the increased RF performance. Studies in [20] reveal the signal power imbalances (PI) between RF channels in the UHF (Ultra-High Frequency) band. In an a typical single-RF 


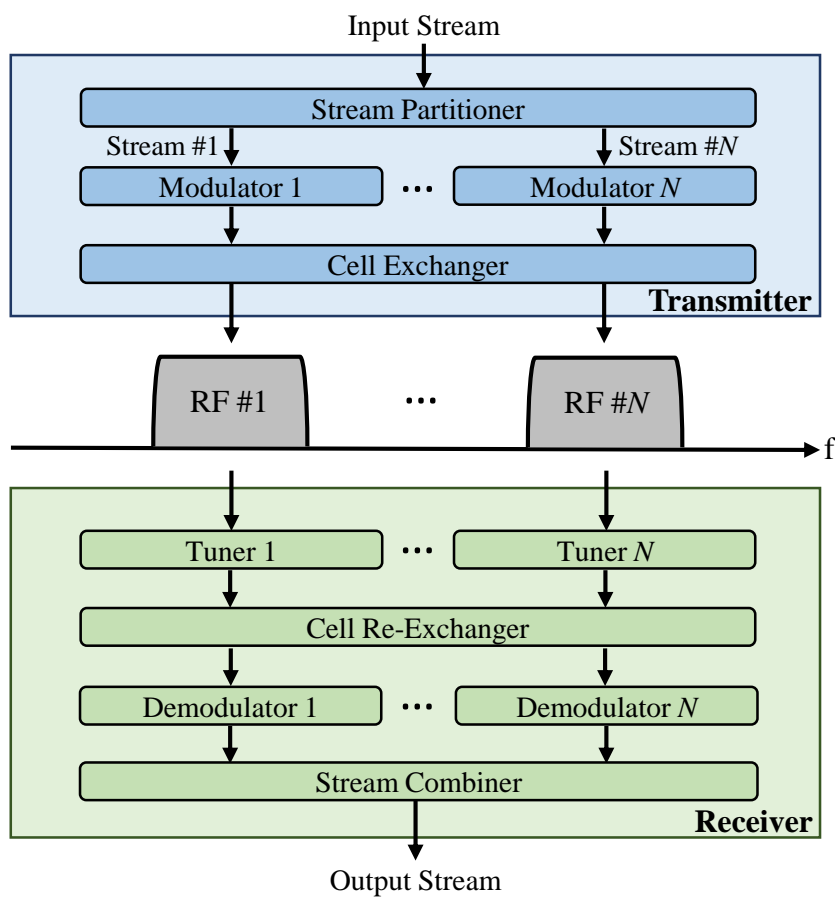

Fig. 6. Channel Bonding concept extended to $N$ RF channels.

transmission, the reception of the complete delivered set of services is limited by the RF channel with the lowest SNR. If one of the RF channels cannot be decoded, the services carried within are lost, regardless of better conditions of the other channels. With a cell exchanger, all services are received with a global SNR corresponding to an averaged SNR among the RF channels [21]. As a matter of considering the gain provided by CB or TFS $\left(G_{M u l t i-R F}\right)$, Eq. 2 accounts for the additional SNR margin provided by multi-RF ( $S N R_{\text {Multi-RF }}$ ) over the SNR of the worst received RF channel $\left(S N R_{\text {worst }}\right)$.

$$
G_{\text {Multi-RF }}[d B]=S N R_{\text {Multi-RF }}-S N R_{\text {worst }}
$$

Additional gains can be exploited by the combination of rotated constellation and multi-RF techniques. In such case, it is desirable that each component is transmitted in a different RF channel. In this paper, we apply the CIL only to the Q component, and the design becomes different depending on the number of RF channels currently used in the transmission. It is based on the CIL specified in the DVB-NGH (Next-Generation Handheld) standard [2]. The Q components are shifted from RF channel to RF channel as shown in Fig. 7.

\section{Performance Evaluation}

\section{A. Non-Uniform Rotated Constellations Gain}

Fig. 8 depicts the rotation gain, i.e. the improvement in the required SNR due to the constellation rotation. We consider the NURCs optimized with the OAR method, from 16QAM to 256QAM, and the QPSK constellation (without any possible optimization). CRs from ATSC 3.0 are evaluated. The highest rotation gain is obtained for low-order constellations and high CRs. The additional diversity introduced by RCs improves

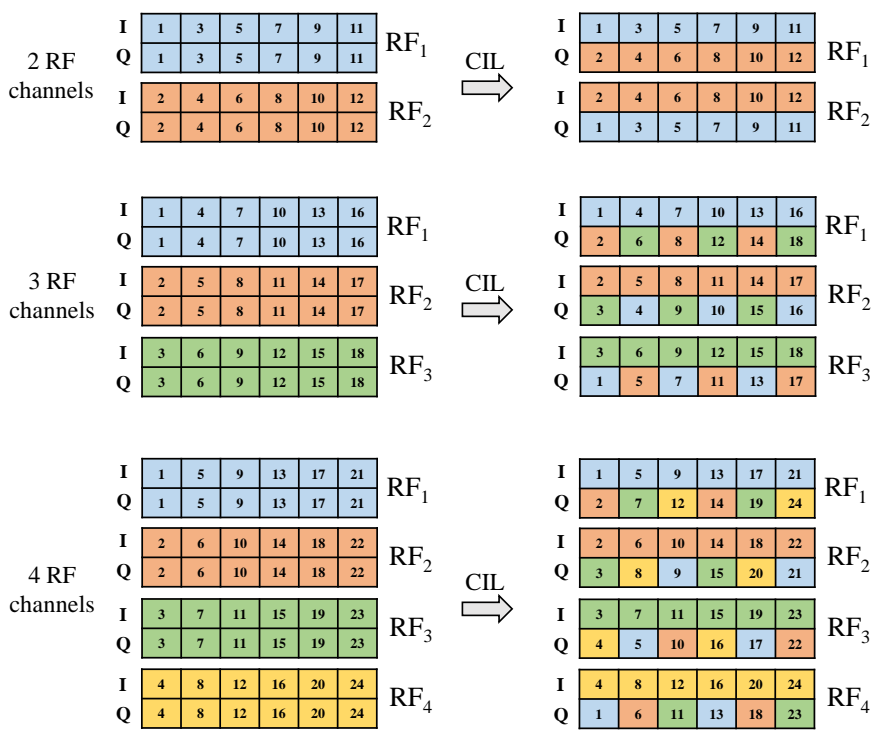

Fig. 7. Component Interleaver designed for multi-RF techniques, using 2, 3 or 4 RF channels. It is only applied to the Q component. The I component keeps the same distribution.

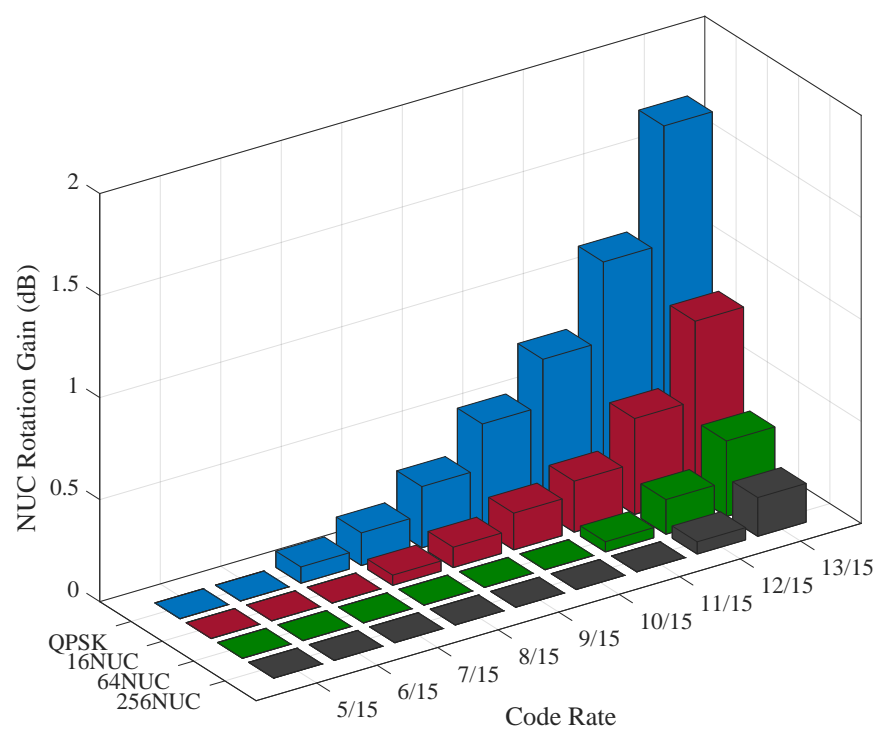

Fig. 8. Rotation gain (dB) of 2D-NURCs designed with OAR for i.i.d. Rayleigh channel, when a Q-delay is applied. CRs from 5/15 to $13 / 15$.

the performance for higher CRs, whereas for lower CRs it is preferable to rely on the error-correction capabilities of the FEC code [15]. In this case, a maximum gain of 1.7 $\mathrm{dB}$ is obtained using QPSK 13/15. It should be noted that the required SNR with OAR and 16NURC is $0.2 \mathrm{~dB}$ and $0.15 \mathrm{~dB}$ higher than with OBR, using CRs of $9 / 15$ and $10 / 15$ respectively. This is due to the significant improvement obtained in the optimization process for SNRs from 9 to 11 $\mathrm{dB}$, range where these particular modes work.

\section{B. Case Study: Non-Uniform Rotated Constellations with Multiple RF Channels}

This section focuses on the gain of rotated constellations when making use of inter-RF frequency diversity, transmitting 


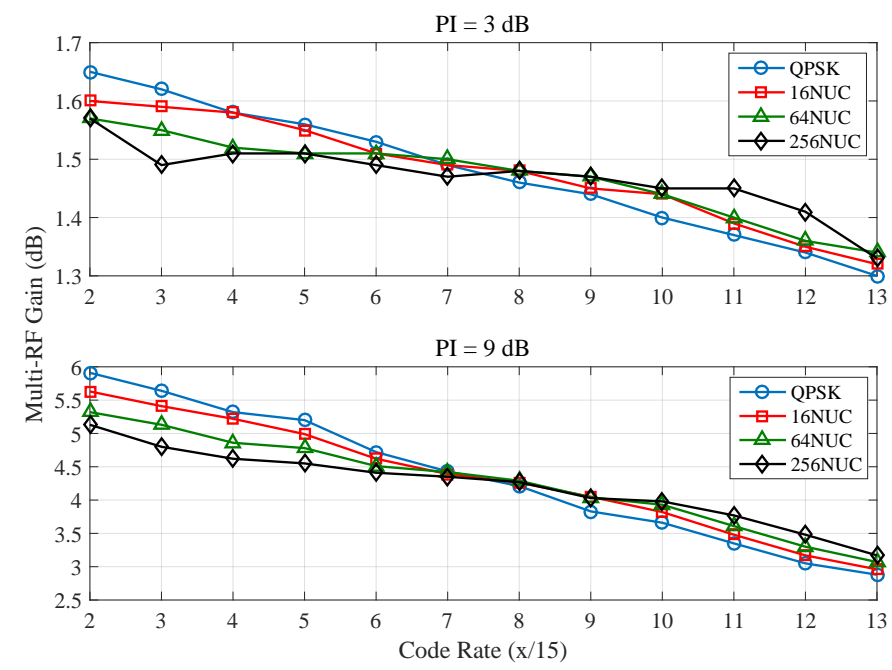

Fig. 9. Multi-RF Gain (dB) for two RF channels, depending on the CR and modulation, iid Rayleigh channel. Two different scenarios are studied, i.e. PI of 3 and $9 \mathrm{~dB}$.

the I/Q components of each rotated symbol in different RF channels. First, we only consider 2 RF channels. The study is extended to $4 \mathrm{RF}$ channels. In order to model these RF channels, two different power imbalances are defined, i.e. 3 and $9 \mathrm{~dB}$. We consider $3 \mathrm{~dB}$ to simulate that the RF channels are close in the same frequency range, and transmitted under similar channel conditions. An imbalance of $9 \mathrm{~dB}$ is also studied as a worst-case scenario. Studies in [20] reveal that it is possible to find imbalances up to $10 \mathrm{~dB}$ in certain locations.

1) Gain with 2 RF Channels: Fig. 9 depicts the multi-RF gain $\left(G_{m u l t i-R F}\right)$ obtained for all NUCs, optimized for a single $\mathrm{RF}$ transmission, and the two considered scenarios (imbalances of 3 and $9 \mathrm{~dB}$ ). The multi-RF gain is calculated using Eq. 2 . It should be noted that the higher the PI between RF channels, the larger the gain. The gain also depends on two additional parameters: CR and order of constellation. Regardless of the PI between RF channels and without applying any rotation to the constellation, the highest gains are for very robust LDPC codes (low CR) with a low-order modulation, e.g. QPSK, obtaining a maximum gain of $5.9 \mathrm{~dB}$. As a half-way point, CRs such as $7 / 15$ or $8 / 15$ provide a gain which is approximately the half of the PI between RF channels. From this point, we assume the worst considered scenario ( $9 \mathrm{~dB}$ of imbalance) for further results.

Fig. 10 shows the rotation gain $\left(G_{R O T}\right)$ of NURCs when using multi-RF techniques. As occurred with a single RF channel, the additional diversity introduced by RCs improves the SNR performance only for high CRs, but the range of CRs with additional gain is increased. In particular, there are two more combinations where a rotation gain can be achieved in multi-RF scenarios. These two combinations are QPSK with CRs $5 / 15$ and 6/15, with rotation angles of $10.5^{\circ}$ and $12^{\circ}$ respectively. Moreover, the obtained gains become significantly higher. The maximum SNR gain, obtained with a QPSK constellation and CR 13/15, is $3.9 \mathrm{~dB}$, which implies an increase of $2.2 \mathrm{~dB}$ compared to the single-RF case.

Fig. 11 depicts the total gain (continuous lines) achieved

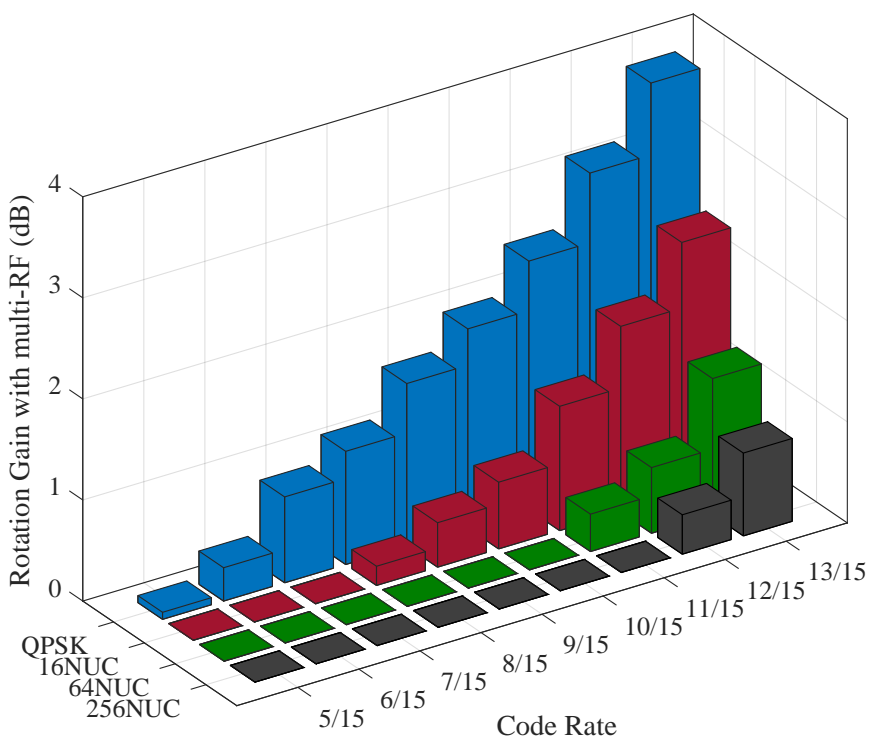

Fig. 10. Rotation SNR gain $\left(G_{R O T}\right)$ of NURCs with multi-RF techniques and $2 \mathrm{RF}$ channels, for i.i.d Rayleigh channel and PI of $9 \mathrm{~dB}$.

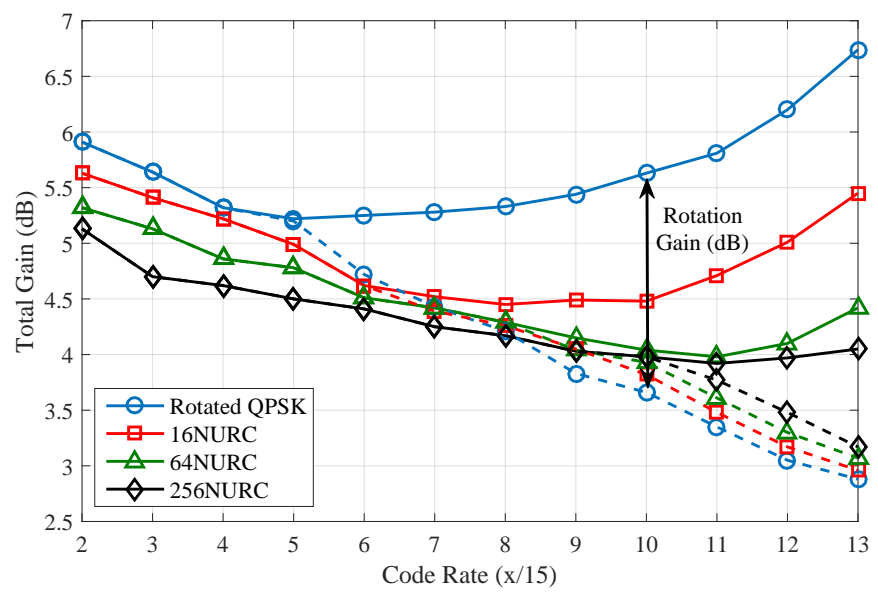

Fig. 11. Total gain $\left(G_{T}\right)$ of NURCs with multi-RF techniques, for i.i.d Rayleigh channel and PI of $9 \mathrm{~dB}$ between the $2 \mathrm{RF}$ channels. Dashed lines represent the multi-RF gain $\left(G_{m u l t i-R F}\right)$, without rotation.

when combining both the rotation and multi-RF procedures $\left(G_{T}=G_{m u l t i-R F}+G_{R O T}\right)$, with a PI of $9 \mathrm{~dB}$, compared to the non-rotated case (dashed lines). In contrast to non-rotated constellations, the highest gain is achieved with the largest possible CR, when using the QPSK modulation. In this case, the SNR gain obtained is up to $6.7 \mathrm{~dB}$, from a total of $9 \mathrm{~dB}$ of imbalance. With higher-order constellations, even though the rotation provides a considerable gain, low CRs remain as the better option. The total gain $G_{T}$ is always over $4 \mathrm{~dB}$ for all cases. Thanks to the SNR averaging combined with the rotation, almost the half of power imbalance between RF channels could be recovered, for any configuration.

2) Gain with 4 RF Channels: In this section, the potential gain of NURCs with SNR averaging over 4 RF channels is analyzed. We consider 3 different scenarios, depending on the number of channels with a very low SNR compared to the best RF channel: when $1 / 4,2 / 4$ or $3 / 4$ RF channels are transmitted 


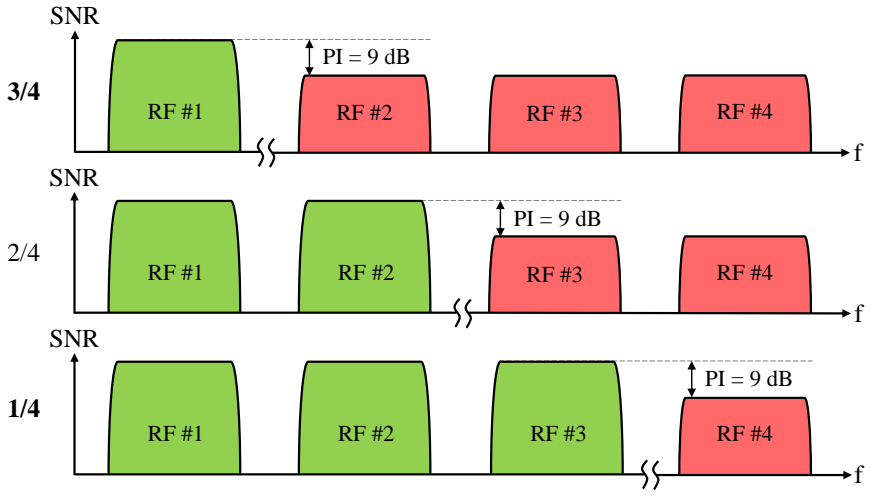

Fig. 12. Three considered scenarios where multi-RF with SNR averaging and $4 \mathrm{RF}$ channels can be potentially beneficial. PI is fixed to $9 \mathrm{~dB}$.

with a PI of $9 \mathrm{~dB}$, as shown in Fig. 12. From the diversity point of view, the case of $2 / 4 \mathrm{RF}$ channels is analog to $1 / 2$ with 2 RF channels, studied in previous section.

From the two possible remaining scenarios, the most relevant result can be derived from the $3 / 4$ case, i.e. when $3 \mathrm{RF}$ channels present a low performance compared to the best one. Thanks to multi-RF tecnhiques and SNR averaging, the minimum required SNR can be drastically reduced, at the expense of requiring a higher SNR in the RF channel with the best performance. When rotated constellations are also employed, the SNR gain becomes higher especially with high CRs and low-order constellations, as occurred with 2 RF channels (see Fig. 13). For instance, with QPSK 13/15, a total gain of $3.8 \mathrm{~dB}$ is achieved in the 3 worst RF channels. Reducing the SNR performance in these 3 channels implies an increase of $5.1 \mathrm{~dB}$ in RF1. As expected, the SNR gain is lower than the $6.7 \mathrm{~dB}$ obtained with $2 \mathrm{RF}$ channels (equivalent to $2 / 4$ ).

On the other hand, with $1 / 4$ a single RF channel is transmitted with a SNR imbalance compared to the rest of channels. In this case, $\mathrm{CB}$ provides very-high gains, especially with low CRs. Without rotation, the maximum SNR gain obtained ranges between 7.5 and $7.8 \mathrm{~dB}$ (the maximum possible gain is $9 \mathrm{~dB}$ ), regardless of the constellation, see Fig. 14. When using NURCs, the SNR range is increased (from $4 / 15$ to $13 / 15$ ), and the rotation gain becomes slightly higher, up to $3.2 \mathrm{~dB}$. Using QPSK 13/15, the total gain is $8.7 \mathrm{~dB}$, which means that the $\mathrm{RF}$ channel with poor performance is almost fully recovered, as long as the SNR of the rest of channels is maintained.

3) Gain with Erasures: As an additional result, in this section we evaluate the potential gains when the RF channels present co-channel interferences that are simulated as erasures [22]. With erasures, there is a direct relationship between $\mathrm{CR}$ and the quality of the channels, which can be explained by considering the amount of erased information symbols during a co-channel interfered transmission. In other words, the presence of erasures limits the maximum CR for which error-free communication is possible. A $25 \%, 50 \%$ and $75 \%$ of erasures require CRs lower than $3 / 4,1 / 2$ and $1 / 4$ respectively in order to repair the loss of information.

Fig. 15 depicts the minimum SNR required at the receiver when using 4 different RF channels, with SNR averaging

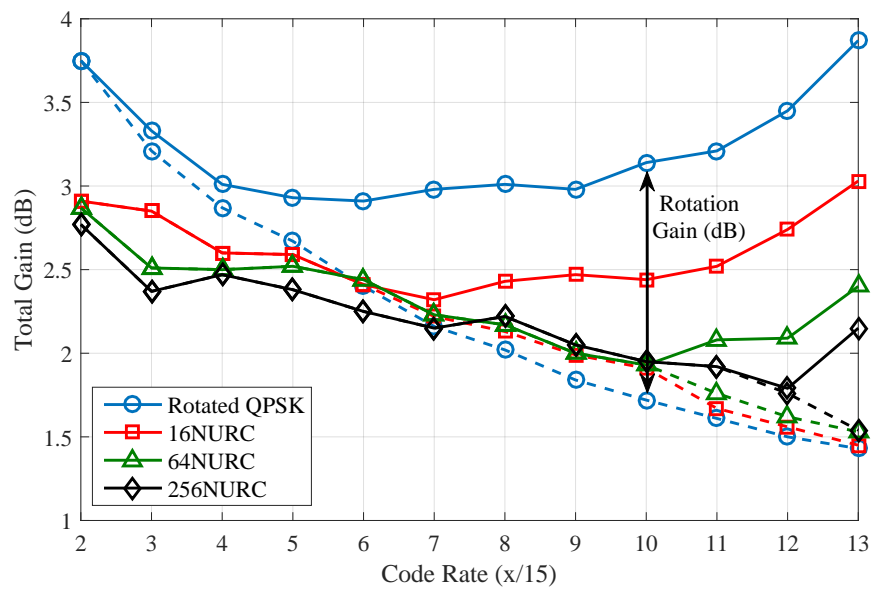

Fig. 13. Total gain $\left(G_{T}\right)$ of NURCs with multi-RF and 4 RF channels, for i.i.d Rayleigh channel and 3/4 RF channels under worse channel conditions. Dashed lines represent the multi-RF gain $\left(G_{C B}\right)$, without rotation.

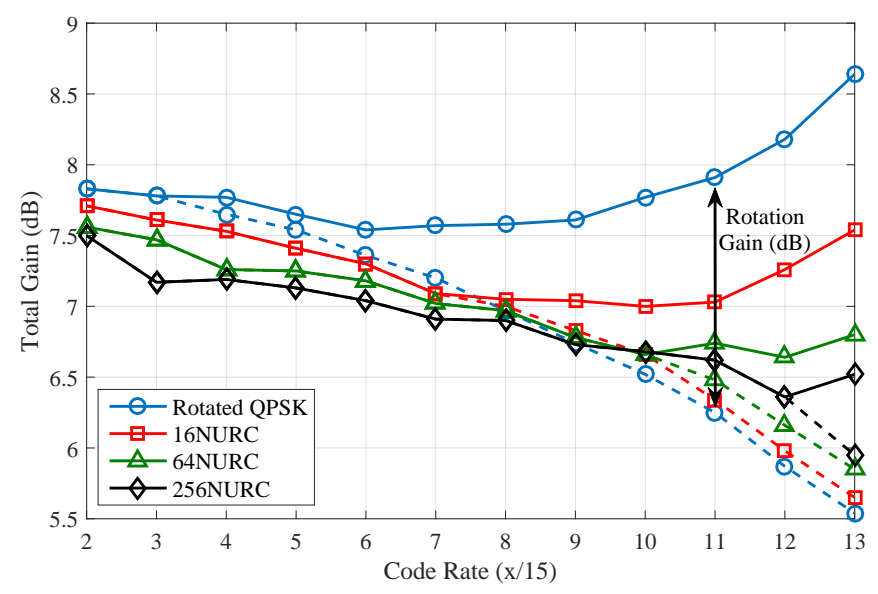

Fig. 14. Total gain $\left(G_{T}\right)$ of NURCs with with multi-RF and 4 RF channels, for i.i.d Rayleigh channel and 1/4 RF channels under worse channel conditions. Dashed lines represent the multi-RF gain $\left(G_{C B}\right)$, without rotation.

and the presence of erasures in 1, 2 or 3 channels, without rotation (dashed lines). As mentioned before, the higher the $\mathrm{CR}$, the larger the SNR required. With erasures and high CRs the SNR tends to infinite, which makes the demodulation process impossible. The use of RC (continuous lines) allows extending the SNR range where it is possible to recover the desired capacity. Whereas it was not possible to recover the capacity lost by the imbalance on RF2 with CRs higher than $1 / 2$ for QPSK, with $\mathrm{RC}$ it is possible to recover the lost RF channel for higher SNR values, at the expense of a higher SNR requirement. With RCs, a $25 \%, 50 \%$ and $75 \%$ of erasures allow to use CRs lower than $13 / 15,9 / 15$ and $6 / 15$, respectively.

\section{CONCLUSION}

In this paper, we have first described the optimization process of Non-Uniform Rotated Constellations (NURC) and analyzed the performance in a single-RF transmission. Even though the demapping complexity is not increased, the SNR gain of NURCs in this case is not significant. The highest rotation gain is obtained for low-order constellations and high 


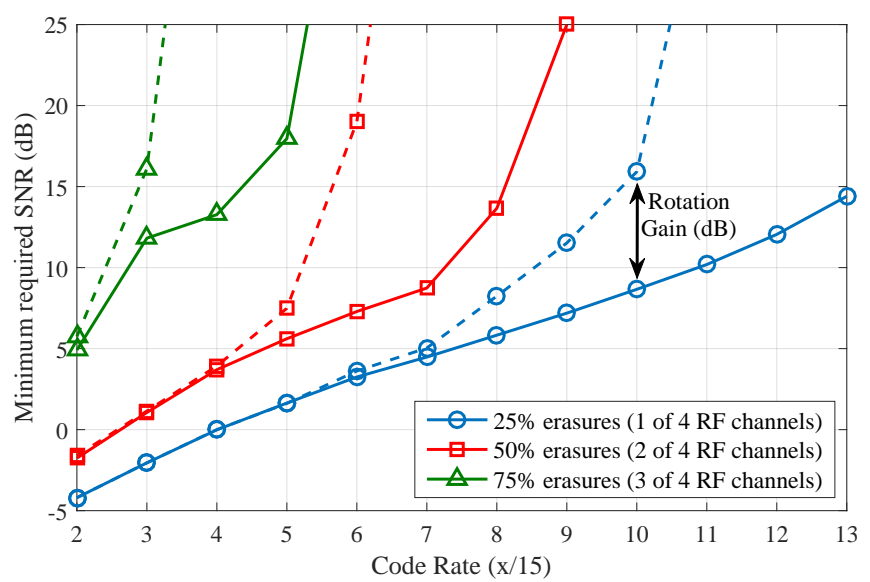

Fig. 15. Minimum required SNR of rotated QPSK with multi-RF and erasures in the $25 \%, 50 \%$ or $75 \%$ of the $4 \mathrm{RF}$ channels. Dashed lines represent the SNR required without rotation.

CRs, obtaining a maximum of $1.7 \mathrm{~dB}$ using QPSK 13/15. However, with multi-RF techniques such as Channel Bonding (CB) and Time-Frequency Slicing (TFS), the SNR gain is drastically increased, since I and Q components are transmitted in different RF channels. Hence, we have provided the multiRF gain of NUCs with and without rotation. Without rotating NUCs, the higher the PI between RF channels, the larger the gain. The gain also depends on the $\mathrm{CR}$ and order of constellation. Regardless of the PI between RF channels, the highest gains are for very robust LDPC codes (low CR) with a low-order modulation. When applying an additional rotation, the highest gain is achieved with the largest possible CR, when using the QPSK modulation. In this case, the SNR gain obtained is up to $6.7 \mathrm{~dB}$, with a PI of $9 \mathrm{~dB}$. When extending the results to $4 \mathrm{RF}$ channels, we have considered two additional scenarios: $3 / 4$ and 1/4 RF channels with poor performance. The most important result derives from the second scenario, where the RF channel with poor performance can be almost fully recovered using QPSK 13/15. Additional results to these scenarios have been provided, when the RF channels present co-channel interferences. A $25 \%, 50 \%$ and $75 \%$ of erasures require CRs lower than $3 / 4,1 / 2$ and $1 / 4$ respectively in order to repair the loss of information. With an additional rotation, the maximum $\mathrm{CR}$ is increased to $13 / 15,9 / 15$ and $6 / 15$, respectively.

\section{REFERENCES}

[1] L. Michael and D. Gomez-Barquero, Bit-Interleaved Coding and Modulation (BICM) for ATSC 3.0, IEEE Trans. Broadcast., vol. 62, no. 1, pp. 181-188, March 2016.

[2] D. Gomez-Barquero, C. Douillard, P. Moss, and V. Mignone, DVB-NGH: The Next Generation of Digital Broadcast Services to Handheld Devices, IEEE Transactions on Broadcasting, vol. 60, no. 2, pp. 246-257, June 2014.

[3] T. Ikeda, Transmission System for ISDB-T $T_{S B}$ (Digital Terrestrial Sound Broadcasting), Procedings of the IEEE, vol. 94, no. 1, pp. 257-260, Jan. 2006.

[4] U. Ladebusch and C. A. Liss, Terrestrial DVB (DVB-T): A Broadcast Technology for Stationary Portable and Mobile Use, Procedings of the IEEE, vol. 94, no. 1, pp. 183-193, Jan. 2006.
[5] I. Eizmendi, et al., DVB-T2: The Second Generation of Terrestrial Digital Video Broadcasting System, IEEE Transactions on Broadcasting, vol. 60, no. 2, pp. 258-271, June 2014.

[6] N. Loghin et al., Non-Uniform Constellations for ATSC 3.0, IEEE Trans. Broadcast., vol. 62, no. 1, pp. 197-203, March 2016.

[7] L. Fay, et al., An Overview of the ATSC 3.0 Physical Layer Specification, IEEE Trans. Broadcast., vol.62, no. 1, pp. 159-171, March 2016.

[8] C. Douillard and C. A. Nour, Bit-Interleaved Coded Modulation in NextGeneration Mobile Broadcast Standard DVB-NGH, in Next Generation Mobile Broadcasting, D. Gomez-Barquero (ed.), CRC Press, 2013, pp. 321-354.

[9] P. Xue, K. Bae, K. Kim, and H. Yang, Best Candidates Based SoftDemapper for Rotated M-QAM Constellation, IEEE 11th Consumer Communications and Networking Conference (CCNC), Las Vegas, USA, Jan. 2014.

[10] G. J. Foschini and S. B. Weinstein, Optimization of Two-Dimensional Signal-Constellations in the Presence of Gaussian Noise, IEEE Transactions on Communications, vol. 22, no. 6, pp. 28-38, Jan. 1974.

[11] J. Stott, CM and BICM limits for rectangular constellations, BBC Research \& Development White Paper WHP 257, Aug. 2013.

[12] J. Zöllner and N. Loghin, Optimization of High-order Non-uniform QAM Constellations, IEEE International Symposium on Broadband Multimedia Systems and Broadcasting (BMSB), London, UK, June 2013.

[13] B. Mouhouche, D. Ansorregui, and A. Mourad, High Order NonUniform Constellations for broadcasting UHDTV, IEEE Wireless Communications and Networking Conference, Istambul, Turkey, April 2014.

[14] M. Fuentes, D. Vargas and D. Gomez-Barquero, Low-Complexity Demapping Algorithm for Two-Dimensional Non-Uniform Constellations, IEEE Transactions on Broadcasting, June 2016.

[15] D. Gozalvez, J. J. Gimenez, D. Gomez-Barquero, and N. Cardona, Rotated Constellations for Improved Time and Frequency Diversity in DVB-NGH, IEEE Transactions on Broadcasting, vol. 59, no. 2, pp. 298305, June 2013

[16] L. Stadelmeier, D. Schneider, J. Zöllner, and J. J. Gimenez, Channel Bonding for ATSC 3.0, IEEE Trans. Broadcast., vol. 62, no. 1, pp. 289297, March 2016.

[17] J. J. Gimenez, E. Stare, S. Bergsmark, D. Gomez-Barquero, Time Frequency Slicing for Future Digital Terrestrial Broadcasting Networks, IEEE Transactions on Broadcasting, vol. 60, no. 2, pp. 227-238, June 2014.

[18] K.-J. Kim, et al., Low-Density Parity-Check Codes for ATSC 3.0, IEEE Trans. Broadcast., vol.62, no. 1, pp. 189-196, March 2016.

[19] J. A. Nelder, R. Mead, A simplex method for function minimization, Computer Journal, vol.7, pp. 308-313, 1965.

[20] J. J. Gimenez, D. Gozalvez, D. Gomez-Barquero, and N. Cardona, Statistical Model of Signal Strength Imbalance Between RF Channels in DTT Network, Electronic Letters, vol. 48, no. 12, pp. 731-732, June 2012.

[21] M. Makni, J. Robert, E. Stare, Performance analysis of time frequency slicing, 14th ITG Conference on Electronic Media Technology (CEMT), Dortmund, Germany, March 2011

[22] ETSI TS 102831 V1.2.1, Digital Video Broadcasting (DVB); Implementation guidelines for a second generation digital terrestrial television broadcasting system (DVB-T2), Aug. 2012.

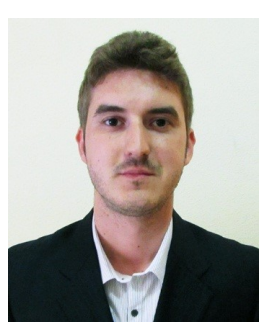

Manuel Fuentes received his M.Sc. degree in telecommunication engineering and a second M.Sc. degree in technologies, systems and communication networks from the Universitat Politecnica de Valencia, Spain, in 2012 and 2013, respectively. Currently, he is pursuing a Ph.D. degree in telecommunications at the Institute of Telecommunications and Multimedia Applications (iTEAM), where he is working since 2012. He participated in several R\&D projects where his research interests were focused on protection ratio measurements and network planning activities between digital broadcasting and 4G (LTE) technologies. He has been a guest researcher at the Vienna University of Technology, Austria, in 2016. He is also a current member of the ATSC forum, where he has contributed several times to the ATSC 3.0 standardization process. His main research interests include innovative techniques in bit-interleaved coding and modulation systems, such as non-uniform constellations or signal space diversity techniques, and multi-antenna communications. 


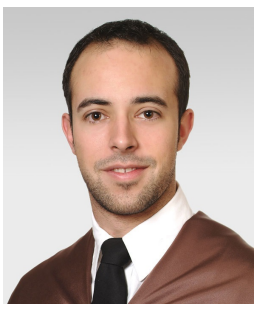

Jordi Joan Gimenez received a M.Sc. degree in Telecomunications engineering (2010), a M. Sc. degree in Technologies, Systems and Communication Networks (2011), and a Ph. D. in Telecommunication (2015) from the Universitat Politcnica de Valncia. During his doctoral studies, he was a Guest researcher at the Royal Institute of Technology (Stockholm, Sweden), and at Teracom AB (Stockholm, Sweden), the Swedish Digital Terrestrial TV operator. He has been a Post-Doctoral Guest Researcher at the Institut für Rundfunktechnik (Munich, Germany) in 2015. Dr. Gimenez is a researcher at the Institute of Telecommunications and Multimedia Applications (iTEAM-UPV), within the group working on next generation cellular and television broadcasting technologies. He has participated in the standardization process of the next-generation handheld standard DVB-NGH, as well as in the DVB technical group working on the assessment of transmission technologies for the next generation terrestrial broadcasting (DVB TM-T MIMO Study Mission). Part of the results of his research has also been presented in the ATSC 3.0 standardization process, within the Waveform Ad-Hoc group. His main research interests include the network planning of next-generation terrestrial broadcast networks as well the characterization and modelling of propagation in the broadcast frequency bands.

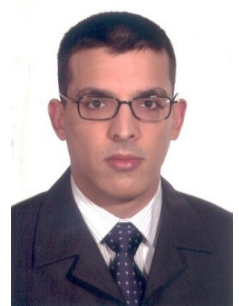

David Gómez-Barquero received the double M.Sc. degrees in telecommunications engineering from the Universitat Politecnica de Valencia (UPV), Spain, and the University of Gävle, Sweden, in 2004, and the $\mathrm{Ph} . \mathrm{D}$. degree in telecommunications from the UPV in 2009. He is a Senior Researcher (Ramon Cajal Fellow) with the Institute of Telecommunications and Multimedia Applications, UPV, where he leads a research group working on next generation cellular and television broadcasting technologies. He hold visiting research appointments at Ericsson Eurolab, Germany, the Royal Institute of Technology, Sweden, the University of Turku, Finland, the Technical University of Braunschweig, Germany, the Fraunhofer Heinrich Hertz Institute, Germany, the Sergio Arboleda University of Bogota, Colombia, and the New Jersey Institute of Technology, Newark, NJ, USA. Since 2008, he has been actively participating in the European Digital Television Standardization Forum DVB in different topics such as upper layer forward error correction, DVB-T2, T2-Lite, and DVB-NGH. In 2013, he joined the U.S. Digital Television Standardization Forum ATSC to work on ATSC 3.0, where he is the Vice-Chairman of the Modulation and Coding Ad-Hoc Group. He is the Editor of the book entitled Next Generation Mobile Broadcasting (CRC Press) and an Associate Editor of the IEEE TRANSACTIONS ON BROADCASTING. 